\title{
Radical resection of solitary tracheal extramedullary plasmacytoma under non-intubated anesthesia: a case report
}

\author{
Yinjun $\mathrm{Li}^{1,2,3,4 \#}$, Yu Jiang ${ }^{1,2,3,4,5 \#}$, Zixuan $\mathrm{Su}^{1,2,3,4,5 \#}$, Hengrui Liang ${ }^{1,2,3,4}$, Jianxing $\mathrm{He}^{1,2,3,4}$, Shuben $\mathrm{Li}^{1,2,3,4}$ \\ ${ }^{1}$ Department of Thoracic Surgery and Oncology, The First Affiliated Hospital of Guangzhou Medical University, Guangzhou, China; ${ }^{2}$ State Key \\ Laboratory of Respiratory Disease, Guangzhou, China; ${ }^{3}$ National Clinical Research Center for Respiratory Disease, Guangzhou, China; ${ }^{4}$ Guangzhou \\ Institute of Respiratory Health, Guangzhou, China; ${ }^{5}$ Nanshan School, Guangzhou Medical University, Guangzhou, China \\ \#These authors contributed equally to this work. \\ Correspondence to: Shuben Li, MD. Department of Thoracic Surgery, the First Affiliated Hospital of Guangzhou Medical University, Guangzhou \\ 510120, China; China State Key Laboratory of Respiratory Disease \& National Clinical Research Center for Respiratory Disease, Guangzhou \\ 510120, China. Email: 13500030280@163.com; Jianxing He, MD, PhD, FACS, FRCS, AATS active member, ESTS member. Department of \\ Thoracic Surgery, the First Affiliated Hospital of Guangzhou Medical University; China State Key Laboratory of Respiratory Disease \& National \\ Clinical Research Center for Respiratory Disease, Guangzhou 510120, China. Email: drjianxing.he@gmail.com.
}

\begin{abstract}
Extramedullary plasmacytoma (EMP) is an uncommon monoclonal plasma cell malignancy that arises outside of the bone marrow. Rarely, EMPs can occur in the trachea, resulting in severe respiratory distress. Due to a small number of cases, the optimal management of tracheal EMP remains a topic of debate. Here, we report a rare case of solitary tracheal EMP causing symptoms of cough, sputum, paroxysmal nocturnal dyspnea, and progressive exertional dyspnea in a 65-year-old male patient. Computerized tomography and fibro bronchoscopy indicated a pedicled nodular mass on the anterior tracheal wall obstructing over $95 \%$ of the lumen. The patient was soon successfully managed with partial tracheal resection and reconstruction surgery under non-intubated anesthesia and was diagnosed as EMP by histopathology of the resected mass. Additional laboratory tests excluded the diagnosis of multiple myeloma (MM). There are no signs of recurrence after 6 months of follow-up. Although traditional intubated anesthesia with single-lung mechanical ventilation has been widely applied to radical surgery for tracheal tumors, it is associated with a higher incidence of intubation-related complications and thus prolongs the surgical procedure and postoperative recovery. In this article, we reported the application of tracheal resection and reconstruction under non-intubated anesthesia for the treatment of tracheal EMP, which was proved to be feasible and safe. Non-intubated anesthesia for tracheal resection and reconstruction is likely to be an alternative minimally invasive option for patients with tracheal EMP involving central airways.
\end{abstract}

Keywords: Extramedullary plasmacytoma (EMP); tracheal tumor; non-intubated anesthesia; reconstruction; case report

Submitted Apr 11, 2021. Accepted for publication Jun 20, 2021.

doi: 10.21037/atm-21-1786

View this article at: https://dx.doi.org/10.21037/atm-21-1786

\section{Introduction}

Extramedullary plasmacytoma (EMP) is a rare tumor that proliferates from monoclonal plasma cells. It can both manifest as a single lesion and as multiple myeloma $(\mathrm{MM})$. Generally, the head and neck region and upper aerodigestive tract are commonly affected areas, while tracheal involvement is a rare finding (1). Most patients show symptoms related to the location of the mass. Tracheal resection with end-to-end anastomosis is the standard approach, which is also highly challenging due to the perioperative anesthetic management (2). In recent years, non-intubated thoracic surgery has been intensively applied to treating lung diseases due to the potential benefits of a lower incidence of surgery-associated complications 
and quicker postoperative recovery (3). Also, cross-field ventilation avoidance significantly improved the operative view and enhanced the anastomosis (4). To our knowledge, this is the first case report of solitary tracheal EMP, which was successfully managed with partial tracheal resection and reconstruction under non-intubated anesthesia, and it may provide a potential option for the treatment of tracheal EMP. We present the following case in accordance with the CARE reporting checklist (available at https://dx.doi. org/10.21037/atm-21-1786).

\section{Case presentation}

\section{Patients}

A 65 -year-old male patient with a 40-year-smoking history presented with cough, sputum, shortness of breath, paroxysmal nocturnal dyspnea, and progressive exertional dyspnea for over 8 months. He perceived aggravated dyspnea, especially when lying down. The patient was referred to our hospital in October 2020 because of symptom deterioration. All procedures performed in studies involving human participants were in accordance with the ethical standards of the institutional and/or national research committee(s) and with the Helsinki Declaration (as revised in 2013). Written informed consent was obtained from the patient.

\section{Preoperative evaluation}

When neoplasia is suspected, extension assessments including enhanced cranial magnetic resonance imaging (MRI), general bone scanning, and enhanced abdominal computed tomography (CT) were utilized to exclude distant tumor metastasis. Also, we assessed the cardiorespiratory function, pulmonary ventilation function, and coronary CT to evaluate whether tracheal resection and reconstruction is safe for this patient. As the cost of positron emission tomography-computed tomography (PET-CT) is not included in China National Medical Insurance, we did not arrange a PET-CT examination for the patient considering his economic condition.

No noticeable abnormality was found on physical examination and routine laboratory tests. Chest CT identified a soft tissue mass in the upper trachea without invading the lateral wall (Figure 1A). Fibro bronchoscopy confirmed a pedicled nodular mass measuring about $2.0 \times 1.5 \times 0.8 \mathrm{~cm}$ from the upper tracheal anterior wall, $4 \mathrm{~cm}$ below the glottis, and $5.5 \mathrm{~cm}$ above the carina. It can swing up and down with breathing, occluding over $95 \%$ of the tracheal lumen (Figure 1B). Notably, the tumor leaves a space of about $0.8 \times 0.6 \mathrm{~cm}$ in the airway when inhaling, causing an illusion that the lung ventilation function test remains within the normal limits, suggesting a certain degree of concealment of tracheal tumors. Since sampling error is one of the limitations of biopsy, biopsy should be performed on multiple tissues from the tracheal tumor. Regrettably, considering the tumor location and the high risk and severity of asphyxia arisen from tumor shedding or hemorrhage during the biopsy, we did not conduct the preoperative pathological biopsy.

\section{Anesthesia}

Tracheal resection and reconstruction under non-intubated anesthesia was performed using intravenous anesthesia. While utilizing a mask to supply oxygen and remove nitrogen, $1.8 \mu \mathrm{g} / \mathrm{mL}$ propofol was given intravenously by target-controlled infusion (TCI). When sufficient sedation was achieved, a laryngeal mask airway (LMA) was placed with $2 \mathrm{mg}$ intravenous infusion of muscle relaxant, delivering $40 \%$ oxygen at a rate of $3.5 \mathrm{~mL} / \mathrm{min}$. Meanwhile, the anesthesia machine was connected to provide simultaneous intermittent mandatory ventilation (SIMV). Subsequently, intravenous infusion of $1.8 \mu \mathrm{g} / \mathrm{mL}$ propofol via TCI, infusion of remifentanil $0.05 \mu \mathrm{g} / \mathrm{kg} \cdot \mathrm{min}$, together with dexmedetomidine hydrochloride $13 \mathrm{~mL} / \mathrm{h}$ were performed continuously to maintain spontaneous breathing throughout the surgery. During the operation, an intraoperative spectrum analyzer was used to monitor the depth of anesthesia, keeping the bispectral index (BIS) at $40-60$ and the respiratory rate at $12-18$ beats/min according to the monitored parameters. In light of the safety of the whole procedure, devices for endotracheal intubation, thoracoscopic intubation, and mechanical ventilation were also prepared to prevent emergencies like airway obstruction or sudden hemorrhage.

\section{Surgical process}

After thorough preoperative evaluation and preparation, the patient underwent optimized partial tracheal resection and reconstruction surgery under non-intubated anesthesia. The tracheal mass and part of the tracheal tissue were resected at the proximal and distal pre-resection margins of the tumor. The intraoperative frozen-section result showed negative 

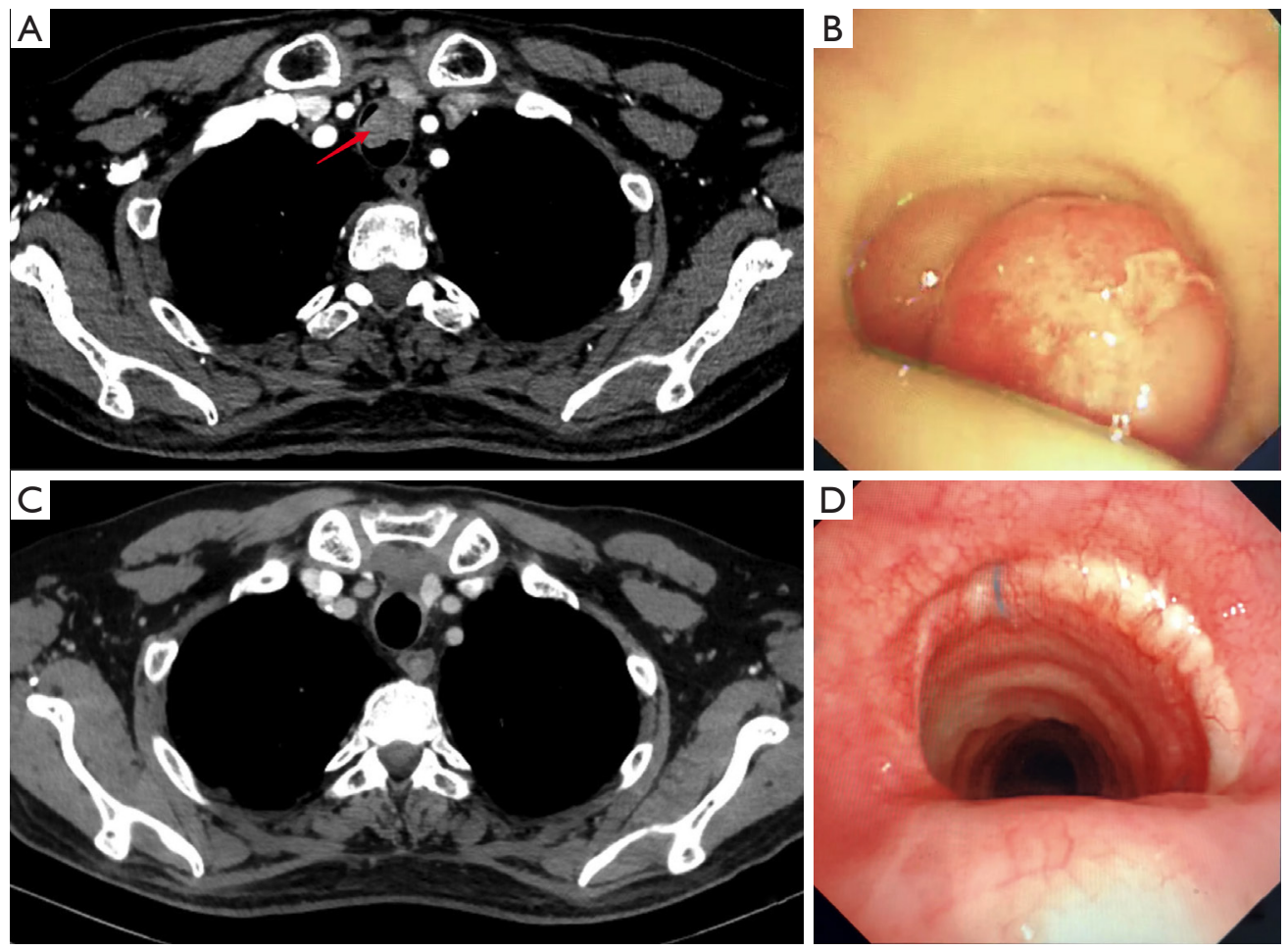

Figure 1 Perioperative evaluation and conditions of the patient. (A) Preoperative chest enhancement CT showed that a soft tissue mass was located inside the upper trachea (arrow). (B) Preoperative fibro bronchoscopy revealed the tumor almost completely obstructed the tracheal lumen. (C) Postoperative CT showed the tumor was completely resected after surgery. (D) Postoperative fibro bronchoscopy showed the anastomoses were patent. No obvious twisting, stenosis, or active bleeding was observed in the trachea. CT, computed tomography.

tumor infiltration at the stumps. After reconstructing the tracheal stump by end-to-end anastomosis (interrupted sutures, 4-0 Vicryl), we embedded the tracheal anastomosis with the sternohyoid muscle. Finally, we placed two prophylactic chin sutures to secure the neck and reduce the extra tension on the anastomosis.

\section{Postoperative conditions}

The patient had no obvious postoperative symptoms like nausea and vomiting but experienced slight pain at the surgical site. Consequently, we provided nonsteroidal antiinflammatory drugs (NSAIDs) and ambroxol to relieve symptoms. The patient had a satisfactory postoperative recovery and was remarkably relieved from the respiratory symptoms without any surgery-related complication (Figure 1C). He was discharged on the fifth postoperative day since he was confirmed ideally healed by bronchoscopy (Figure 1D). During the 6-month follow-up, the patient was in a relatively good physical condition and no sign of tumor recurrence or metastasis was observed.

\section{Postoperative diagnosis}

The paraffin-embedded surgical specimens were pathologically diagnosed as plasmacytoma. The results of immunohistochemical staining were as follow: CD38 (+), CD138 (partial +), CD79a (+), CD20 (-), CD56 (-) and $\lambda$ (partial + ). The MM workup was therefore conducted. The bone marrow aspiration and biopsy showed normal morphology. The negative result of urinary BenceJones protein, serum electrophoresis, or serum protein electrophoresis ruled out the diagnosis of MM and confirmed EMP eventually. Since the resection margins were disease-free, the patient was not managed with postoperative adjuvant radiotherapy or chemotherapy.

\section{Discussion}

Surgery plays an important role in managing tracheal EMP 
and radiotherapy can also be regarded as an alternative first-line therapy (5). In our case, the lesion appeared in the upper trachea without extratracheal propagation. In view of the tumor location, inconvenience of endotracheal intubation, and the non-intubated anesthesia experience in our institution, we opted for tracheal resection and reconstruction via non-intubated anesthesia for the treatment of tracheal EMP which has not yet been reported in the world.

Endotracheal intubation after anesthesia induction together with cross-field ventilation are the traditional option and standard airway management for tracheal/carinal resection and reconstruction, whose safety and applicability have been demonstrated in most clinical cases. However, given that endotracheal intubation may increase the risk of airway edema and stenoses, it also poses a challenge for both surgical and anesthesia teams in terms of anesthetic management, not to mention in some special cases of severe airway stenosis (6-8). What's more, frequent tracheal intubation or cross-field ventilation would greatly affect the surgical field and the suturing process, making it harder for tracheal reconstruction. Also, endotracheal intubation is closely related to difficult anastomosis and trachea ischemia reperfusion, which is not conducive to postoperative recovery (4).

To further improve surgical outcomes, non-intubated thoracic surgery has been applied. Compared with conventional intubated anesthesia with single-lung mechanical ventilation, it represents an advanced strategy to perform surgery based on local anesthesia with spontaneous ventilation, avoiding adverse effects correlated with general anesthesia and one-lung ventilation (9). Several studies have demonstrated that non-intubated thoracic surgery could be successfully applied to various thoracic diseases (10), suggesting it a safe and feasible alternative to traditionally intubated general anesthesia surgery, with potential benefits of a lower rate of complications, faster postoperative recovery, and shorter length of stay $(11,12)$. Also, the non-intubated procedure is performed without or with very little muscle relaxant, which could reduce side effects like diaphragmatic dysfunctions, weakness of upper airway muscles, airway obstruction, and hypoxemia, thereby speeding up the postoperative recovery $(13,14)$. What's more, less trachea damage is associated with less oxidative response and better immune function after surgery, which also contributes to shorter in-hospital stays (15).

Although the safety and feasibility of non-intubated thoracic surgery have been investigated and verified, we still have some concerns about the current research outcomes and study design. Since respiratory tract tumors and stenoses are rare, it is difficult to carry out prospective randomized control trials to determine the safety and effectiveness of non-intubated anesthesia for tracheal/carinal resection and reconstruction. What's more, to avoid perioperative respiratory failure, nonintubated anesthesia is only performed in selected patients. Certain exclusion criteria like (I) obesity (BMI $\geq 25 \mathrm{~kg} / \mathrm{m}^{2}$ ); (II) American Society of Anesthesiologists (ASA) grade of $\geq 3$; (III) cardiopulmonary dysfunction (EF $<50 \%$ and $\mathrm{FEV} 1<60 \%$ predicted value); (IV) severe acute respiratory infection; (V) low blood volume, hemodynamic instability, or coagulopathy; (VI) neurological disorders; (VII) difficult airway management (Mallampati score $\geq 3$ ); (VIII) extensive pleural adhesions; and (IX) long operation time ( $>5$ hours) may affect its application in a larger population (16). Meanwhile, for safety consideration, devices for endotracheal intubation, thoracoscopic intubation, and mechanical ventilation should also be prepared during the surgery procedure. In the case of emergency like (I) $\mathrm{SpO}_{2}<90 \%$; (II) continuous $\mathrm{CO}_{2}$ retention, along with the development of respiratory acidosis; and (III) sudden uncontrollable bleeding, endotracheal intubation or thoracoscopic intubation should be performed immediately (17).

Tracheal EMP is highly sensitive to radiotherapy. A minimum dose of $40 \mathrm{~Gy}$ is recommended based on the National Comprehensive Cancer Network guidelines (18). Even though adjuvant radiotherapy is not recommended for patients who have undergone radical surgical resection with negative margins because there is no evidence that adjuvant chemotherapy can improve the prognosis of patients (1). Tracheal EMP has a relatively favorable prognosis. Previous studies concluded that $70 \%$ of these patients would be disease-free after 10 years and $61 \%$ of patients had no MM or recurrence, whereas $22 \%$ of patients relapsed, and $16 \%$ of patients progressed to MM (19). During the 6-month follow-up period, our patients showed a satisfactory clinical response to surgical intervention without recurrence. Given that the conversion risk is the highest in the first 2 years, longterm follow-up is mandatory (20).

In the present study, we report a rare case of tracheal EMP managed with tracheal resection and reconstruction surgery under non-intubated anesthesia. An easier operation process for surgeons and enhanced recovery for patients may become practicable in the clinic. 


\section{Acknowledgments}

Funding: None.

\section{Footnote}

Reporting Checklist: The authors have completed the CARE reporting checklist. Available at https://dx.doi. org/10.21037/atm-21-1786

Peer Review File: Available at https://dx.doi.org/10.21037/ atm-21-1786

Conflicts of Interest: All authors have completed the ICMJE uniform disclosure form (available at https://dx.doi. org/10.21037/atm-21-1786). JH serves as an Editor-inChief of Annals of Translational Medicine from Jun 2019 to May 2024. The other authors have no conflicts of interest to declare.

Ethical Statement: The authors are accountable for all aspects of the work in ensuring that questions related to the accuracy or integrity of any part of the work are appropriately investigated and resolved. All procedures performed in studies involving human participants were in accordance with the ethical standards of the institutional and/or national research committee(s) and with the Helsinki Declaration (as revised in 2013). Written informed consent was obtained from the patient.

Open Access Statement: This is an Open Access article distributed in accordance with the Creative Commons Attribution-NonCommercial-NoDerivs 4.0 International License (CC BY-NC-ND 4.0), which permits the noncommercial replication and distribution of the article with the strict proviso that no changes or edits are made and the original work is properly cited (including links to both the formal publication through the relevant DOI and the license). See: https://creativecommons.org/licenses/by-nc-nd/4.0/.

\section{References}

1. Soutar R, Lucraft H, Jackson G, et al. Guidelines on the diagnosis and management of solitary plasmacytoma of bone and solitary extramedullary plasmacytoma. $\mathrm{Br} \mathrm{J}$ Haematol 2004;124:717-26.

2. Grillo HC. Development of tracheal surgery: a historical review. Part 1: Techniques of tracheal surgery. Ann Thorac
Surg 2003;75:610-9.

3. Wang ML, Hung MH, Hsu HH, et al. Non-intubated thoracoscopic surgery for lung cancer in patients with impaired pulmonary function. Ann Transl Med 2019;7:40.

4. Liang H, Gonzalez-Rivas D, Zhou Y, et al. Nonintubated Anesthesia for Tracheal/Carinal Resection and Reconstruction. Thorac Surg Clin 2020;30:83-90.

5. Stevic R, Ercegovac M, Stojšić J, et al. Rare tracheal tumor: Solitary plasmacytoma. J Postgrad Med 2018;64:115-8.

6. Marin-Corral J, Dot I, Boguña M, et al. Structural differences in the diaphragm of patients following controlled vs assisted and spontaneous mechanical ventilation. Intensive Care Med 2019;45:488-500.

7. Kelly GT, Faraj R, Zhang Y, et al. Pulmonary Endothelial Mechanical Sensing and Signaling, a Story of Focal Adhesions and Integrins in Ventilator Induced Lung Injury. Front Physiol 2019;10:511.

8. Chitilian HV, Bao X, Mathisen DJ, et al. Anesthesia for Airway Surgery. Thorac Surg Clin 2018;28:249-55.

9. Wen $\mathrm{Y}$, Jiang $\mathrm{Y}$, Liang $\mathrm{H}$, et al. Tubeless video-assisted thoracic surgery for lung cancer: is it ready for prime time? Future Oncol 2020;16:1229-34.

10. Mineo TC, Tamburrini A, Perroni G, et al. 1000 cases of tubeless video-assisted thoracic surgery at the Rome Tor Vergata University. Future Oncol 2016;12:13-8.

11. Liu YJ, Hung MH, Hsu HH, et al. Effects on respiration of nonintubated anesthesia in thoracoscopic surgery under spontaneous ventilation. Ann Transl Med 2015;3:107.

12. Wen Y, Liang H, Qiu G, et al. Non-intubated spontaneous ventilation in video-assisted thoracoscopic surgery: a metaanalysis. Eur J Cardiothorac Surg 2020;57:428-37.

13. Wu S, Liu J, Liang H, et al. Factors influencing the length of stay after mediastinal tumor resection in the setting of an enhanced recovery after surgery (ERAS)-TUBELESS protocol. Ann Transl Med 2020;8:740.

14. Liu J, Li S, Shen J, et al. Non-intubated resection and reconstruction of trachea for the treatment of a mass in the upper trachea. J Thorac Dis 2016;8:594-9.

15. Liu J, Cui F, Pompeo E, et al. The impact of nonintubated versus intubated anaesthesia on early outcomes of video-assisted thoracoscopic anatomical resection in non-small-cell lung cancer: a propensity score matching analysis. Eur J Cardiothorac Surg 2016;50:920-5.

16. He J, Liu J, Zhu C, et al. Expert consensus on tubeless video-assisted thoracoscopic surgery (Guangzhou). J Thorac Dis 2019;11:4101-8.

17. Pompeo E, Mineo D, Rogliani P, et al. Feasibility and results of awake thoracoscopic resection of solitary 
Page 6 of 6

pulmonary nodules. Ann Thorac Surg 2004;78:1761-8.

18. Kumar SK, Callander NS, Alsina M, et al. Multiple Myeloma, Version 3.2017, NCCN Clinical Practice Guidelines in Oncology. J Natl Compr Canc Netw 2017;15:230-69.

19. Reyhan M, Tercan F, Ergin M, et al. Sonographic diagnosis
Li et al. Resection of tracheal EMP via non-intubated anesthesia

of a tracheal extramedullary plasmacytoma. J Ultrasound Med 2005;24:1031-4.

20. Tang RR, Wang Y, Liang CN, et al. Multiple extramedullary plasmacytomas of the trachea and pharyngeal tissue: a case report and literature review. Onco Targets Ther 2019;12:1433-7.

Cite this article as: Li Y, Jiang Y, Su Z, Liang H, He J, Li S. Radical resection of solitary tracheal extramedullary plasmacytoma under non-intubated anesthesia: a case report. Ann Transl Med 2021;9(15):1265. doi: 10.21037/atm-21-1786 